\title{
The Influence of Electron Solid-State Plasma on Attenuation of Longitudinal Sound Waves in a Conductor
}

\author{
Alexander Yushkanov \\ Moscow Regional State University, Moscow, Russia; kaf-tfiz@mgou.ru
}

\begin{abstract}
In the present work the problem of the attenuation of longitudinal sound oscillations in a conducting medium are considered. The proposed approach is based on the dynamic interaction of electron gas with the lattice vibrations. This interaction is manifested in the modification of kinetic equation for electrons. The process is accompanied by generation of an electric field.
\end{abstract}

Key words: longitudinal sound wave; electric field; electrons; kinetic equation; sound attenuation

\section{Introduction}

The question of the impact of the electrons on the sound attenuation in the metal are considered for the first time in the work [1]. Approach to the problem in this work was based on the assumption of the influence of Fermi-surface metal deformation under the action of sound waves on the sound attenuation. Thus the need arose in introducing additional "fictitious" forces. These "fictitious" forces is due to the so-called deformational potential $\lambda[2]$. The precise form of this deformation potential as unknown. Therefore, many calculations of the sound wave attenuation are based on the assumption, that the deformation potential is given, but its exact form is unknown [2].

The process of deformation Fermi-surface is due to the interaction of the electron gas with the lattice. Therefore, it inevitably depends on the characteristics of this interaction. This process may not strictly speaking be considered in static approximation as suggested in [1], [2]. Dynamic and kinetic processes must be considered in the analysis of the formation of a Fermi-surface at the propagation of sound waves in the metal. In the 
present work will be considered the approach to the problem, based on the dynamic (kinetic) interaction of the electron gas with the lattice vibrations.

We will consider the propagation of longitudinal sound in isotropic conductor. The aim of this work is consideration of the question how the conduction electrons and generated electric field effect the process of attenuation of the sound wave. The proposed approach is valid both for metals and for other the conducting media, for example semiconductors.

Some aspects of the problem of propagation and attenuation of sound waves in the metal has been considered in the works [5]-[13].

\section{Statement of the problem and basic equations}

Longitudinal sound wave creates a velocity field in the conductor

$$
\mathbf{u}=\mathbf{u}(\mathbf{r}, t)=\mathbf{u}_{0} e^{i(\mathbf{k r}-\omega t)}, \quad \omega=s_{l} k
$$

Here $s_{l}$ — the speed of longitudinal sound waves, $\mathbf{k}$ - the wave vector, $k$ - the wave number.

We assume that the main role in the scattering of electrons plays the interaction with the lattice. Electron-electron collisions at not too low temperatures do not play a significant role in the kinetics of the electrons (at least in metals). We will therefore neglect them. The kinetic equation with regard of the electron-electron collisions have been discussed in several works, for example in [14]-[16].

Without taking into account the electron-electron collisions kinetic equation for electron distribution function $f$ with the collision integral in $\tau$ approximation has the following form [2], [3]

$$
\frac{\partial f}{\partial t}+\mathbf{v} \frac{\partial f}{\partial \mathbf{r}}+e \mathbf{E} \frac{\partial f}{\partial \mathbf{p}}=\nu\left(f_{e q}-f\right)
$$

In this equation $\mathbf{E}$ - the electric field, $\mathbf{v}$ - the electron velocity, $\nu=$ $1 / \tau$ - the frequency of electron scattering, $\tau$ - the average time between two consecutive cases of scattering of the electron. We assume, that the value of $\nu$ (and $\tau$ ) does not depend on the electron energy and the direction his movements. Carrier relaxation time $\tau$ of the twenty most conductive elemental metals are considered in [17].

The function $f_{e q}$ - locally equilibrium distribution of electrons 


$$
f_{e q}=\left[1+\exp \frac{\mathcal{E}-\mu}{k_{B} T}\right]^{-1} .
$$

Here $\mathcal{E}$ - the energy of the electrons, $\mu$ - chemical potential. We assume that in the absence of a sound wave electron distribution can be considered spherically-symmetric. In this case for electron energy $\mathcal{E}_{0}$ we have

$$
\mathcal{E}_{0}=\frac{m \mathbf{v}^{2}}{2}
$$

Here $m$ - the effective mass of the electron.

The sound wave breaks isotropy locally equilibrium distribution of electrons. This distribution must now be an equilibrium in the coordinate system, at rest relative to the lattice. Because the local velocity of the lattice is $\mathbf{u}$ in this case will be

$$
\mathcal{E}=\frac{m(\mathbf{v}-\mathbf{u})^{2}}{2}
$$

We assume that the velocity $\mathbf{u}$ is much less than the thermal electrons velocity (or Fermi velocity for the case of degenerate Fermi-gas). Then the value of (1.4) can be linearized

$$
\mathcal{E} \simeq \frac{m \mathbf{v}^{2}}{2}-m \mathbf{v u}=\mathcal{E}_{0}-m \mathbf{v u}
$$

Linearization of the chemical potential $\mu(\mathbf{r})$ and can be represented in the form

$$
\mu(\mathbf{r})=\mu_{0}+\delta \mu(\mathbf{r})
$$

Here $\mu_{0}$ - chemical potential in the unperturbed case.

Through appropriate linearization of the equilibrium locally functions $f_{e q}$ we get

$$
f_{e q}=f_{0}-\frac{\partial f_{0}}{\partial \mathcal{E}}(m \mathbf{v u}+\delta \mu), \quad f_{0}=\left[1+\exp \frac{\mathcal{E}_{0}-\mu_{0}}{k_{B} T}\right]^{-1} .
$$

Similarly, in the linear case, the term with the electric field in equation (1.2) takes the following form

$$
e \mathbf{E} \frac{\partial f}{\partial \mathbf{p}} \simeq e \mathbf{E} \mathbf{v} \frac{\partial f_{0}}{\partial \mathcal{E}}
$$


The linearized distribution function has the form [2]

$$
f=f_{0}-\frac{\partial f_{0}}{\partial \mathcal{E}} \psi
$$

Taking into account relations (1.5), (1.6) and (1.7) the kinetic equation (1.2) can be written for the function $\psi$ as follows [2]

$$
i \omega \psi-i \mathbf{v k} \psi+e \mathbf{v E}=-\nu(m \mathbf{v u}+\delta \mu-\psi) .
$$

Thus the deviation of the chemical potential from the equilibrium can be written in the following integral

$$
\delta \mu=-\int \frac{\partial f_{0}}{\partial \mathcal{E}} \psi \frac{2 d^{3} p}{(2 \pi \hbar)^{3}} .
$$

Equation (1.8) can be rewritten in the form

$$
-i \omega \psi+i \mathbf{v k} \psi+\nu \psi-\nu \delta \mu=\mathbf{v}(e \mathbf{E}+\nu m \mathbf{u}) .
$$

The last term in the right-hand side of equation (1.9) corresponds to consideration of the drag effect of electrons by movement of atoms of the lattice at the scattering of electrons by lattice vibrations or defects. It is analogous to the "fictitious" force, introduced in [1] (see also [2]). Note that in this approach this term occurs naturally and does not require any additional assumptions.

Equation (1.9) coincides with the kinetic equation describing the response of the electron on external transverse electric field $\mathbf{E}$, if, instead the field $\mathbf{E}$ to consider the value of $\mathbf{E}+\nu m \mathbf{u} / e$, that is, to replace

$$
\mathbf{E} \rightarrow \mathbf{E}+\frac{\nu m \mathbf{u}}{e}
$$

Since $\mathbf{u} \sim \exp (i \mathbf{k r}-i \omega t)$, then the functions $\psi, \mathbf{E}$ have the same dependence on the coordinates and time, i.e.

$$
\psi \sim \exp (i \mathbf{k r}-i \omega t), \quad \mathbf{E} \sim \exp (i \mathbf{k r}-i \omega t) .
$$

Then the electron current density $\mathbf{j}_{e}$ taking into account (1.10) is determined by the following relation [18]

$$
\mathbf{j}_{e}=\sigma_{l}\left(\mathbf{E}+\frac{\nu m}{e} \mathbf{u}\right)
$$


Here $\sigma_{l}=\sigma_{l}(\mathbf{k}, \omega)$ - longitudinal electrical conductivity of the electron plasma.

The electric field is related with the charge density $\rho_{e}$ of electrons and the lattice charge density $(-e N)$ by ratio

$$
\nabla \mathbf{E}=4 \pi\left(\rho_{e}-e N\right) .
$$

Differentiating this ratio with time we obtaine

$$
\frac{\partial N}{\partial t}=-N \nabla \mathbf{u}, \quad \frac{\partial \rho_{e}}{\partial t}=-N \nabla \mathbf{j}_{e} .
$$

Or taking into account (1.1), (1.10)

$$
-i \omega \nabla \mathbf{E}=-4 \pi \nabla\left(\mathbf{j}_{e}-e N \mathbf{u}\right) .
$$

Therefore

$$
i \omega \mathbf{E}=4 \pi\left(\mathbf{j}_{e}-e N \mathbf{u}\right) .
$$

Using for current density ratio (1.11), after some transformations get

$$
\mathbf{E}\left(4 \pi \sigma_{l}-i \omega\right)=-\frac{4 \pi \sigma_{l} \nu m}{e} \mathbf{u} .
$$

The longitudinal conductivity $\sigma_{l}$ is related to longitudinal dielectric permittivity $\varepsilon_{l}[18]$

$$
\varepsilon_{l}(\mathbf{q}, \omega, \nu)=1+\frac{4 \pi i}{\omega} \sigma_{l}(\mathbf{q}, \omega, \nu) .
$$

Using the ratio (1.13) from equations (1.12) we obtain

$$
\mathbf{E}=-\frac{4 \pi i \sigma_{l} \nu m}{\omega e \varepsilon_{l}} \mathbf{u} .
$$

From the obtained expressions (1.2) and (1.14) we find the density of electron current

$$
\mathbf{j}_{e}=\frac{\nu m}{e}\left(1-\frac{4 \pi i \sigma_{l}}{\omega \varepsilon_{l}}\right) \mathbf{u} .
$$




\section{Attenuation coefficient of sound wave}

The energy flux density carried by longitudinal acoustic wave is equal to $[2]$

$$
I=\frac{\rho_{0} u_{0}^{2} s_{l}}{2}
$$

Here $\rho_{0}$ - the density of the substance.

The attenuation coefficient $\Gamma$ is defined by the following expression

$$
\Gamma=\frac{Q}{I}
$$

Here $Q$ - the energy dissipation density of the sound wave. Dissipation is due to the anharmonicity of the lattice vibrations $Q_{l}$ and interaction of sound waves with the electronic component and generated by the wave electric field $Q_{e}$.

Then the value of $Q$ can be written in the form

$$
Q=Q_{l}+Q_{e}
$$

We are interested in the value $Q_{e}$ that is the dissipation, related with the interaction of sound wave with solid-state plasma.

The value $Q_{e}$ is calculated as [4]

$$
Q_{e}=-\frac{1}{2} \operatorname{Re}\left(\mathbf{F u}^{*}\right) .
$$

The value $\mathbf{F}$ in the relation (2.4) is a force acting on the lattice. It consists of two parts. The first $(-e N \mathbf{E})$ part corresponds to the force acting on a charge from the lattice due to the presence of the electric field E. The presence of the minus sign is due to the electroneutrality of the material. So lattice charge density is opposite to charge density of electrons and equal to $(-e N)$. The second term $\mathbf{F}_{e}$ describes the force acting on the lattice from solid-state electron plasma in the process of scattering of electrons on the lattice.

Thus

$$
\mathbf{F}=-e N \mathbf{E}+\mathbf{F}_{e}
$$

Accordingly, for values $Q_{e}$ we obtain the following expression 


$$
Q_{e}=\frac{1}{2} \operatorname{Re}\left(\left(\mathbf{F}_{e}-e N \mathbf{E}\right) \mathbf{u}^{*}\right)
$$

The value $\mathbf{F}_{e}$ can be calculated as follows

$$
\mathbf{F}_{e}=-\int \nu m(\mathbf{u}-\mathbf{v}) f \frac{2 d^{3} p}{(2 \pi \hbar)^{3}}
$$

Evaluation of the integral in (2.6) gives the following result

$$
\mathbf{F}_{e}=-\nu m N \mathbf{u}+\frac{\nu m \mathbf{j}}{e} .
$$

Let us denote the average speed of the electrons through $\overline{\mathbf{v}}$.

$$
\mathbf{j}=e N \overline{\mathbf{v}}
$$

Then formula (2.7) can be rewritten in the form

$$
\mathbf{F}_{e}=\nu m N(\overline{\mathbf{v}}-\mathbf{u})
$$

Thus the force is proportional to the difference between the average velocity of the electrons in solid-state plasma and displacement velocity of atoms in the sound wave.

Substituting (2.7) into (2.4) and using (1.11) we obtain the following expression for $Q_{e}$

$$
Q_{e}=-\frac{1}{2} \operatorname{Re}\left(\left(-e N+\frac{\nu m \sigma_{l}}{e}\right) \mathbf{E}-\left(\nu m N-\frac{\sigma_{l} \nu^{2} m^{2}}{e^{2}}\right) \mathbf{u}\right) \mathbf{u}^{*} .
$$

The electric field $\mathbf{E}$ is given by (1.4). Note that $\mathbf{u u}^{*}=u_{0}^{2}$. As a result, after some transformations we get

$$
Q_{e}=\frac{\nu^{2} m^{2} u_{0}^{2}}{2 e^{2}} \operatorname{Re}\left[\sigma_{0}-\sigma_{l}+\frac{4 \pi i}{\omega \varepsilon_{l}}\left(\sigma_{0}-\sigma_{l}\right)^{2}\right] .
$$

Here $\sigma_{0}=N e^{2} /(m \nu)$ — static conductivity of an electron solid-state plasma.

Consider the case of small wave numbers. That is, suppose $k \rightarrow 0$. Then, according to (1.1) $\omega \rightarrow 0$. Thus $\sigma_{l} \rightarrow \sigma_{0}$. From the obtained expression for $Q_{e}(2.7)$, we obtain that in this case, $Q_{e} \rightarrow 0$.

In accordance with the expressions (2.2) and (2.3) the sound wave attenuation coefficient $\Gamma$ can be split into two parts 


$$
\Gamma=\Gamma_{l}+\Gamma_{e}, \quad \Gamma_{l}=\frac{Q_{l}}{I}, \quad \Gamma_{e}=\frac{Q_{e}}{I}
$$

We will be interested in the value $\Gamma_{e}$. Taking into account expressions (2.1), (2.8) and (2.9) for the value we obtain the following result

$$
\Gamma_{e}=\frac{\nu^{2} m^{2} u_{0}^{2}}{\rho_{0} s_{l} e^{2}} \operatorname{Re}\left[\sigma_{0}-\sigma_{l}+\frac{4 \pi i}{\omega \varepsilon_{l}}\left(\sigma_{0}-\sigma_{l}\right)^{2}\right] .
$$

Note that the attenuation coefficient of (2.10) tends to zero in the longwave limit, i.e. when $k \rightarrow 0$.

\section{Conclusion}

In this work the influence of electron solid-state plasma on the attenuation of longitudinal sound wave is considered. The approach is equally true for case of degenerate (metal) and for the case of nondegenerate solid-state plasma. The approach is based on the dynamics of the interaction of the electron components with the lattice. This interaction displays itself in the scattering of electrons on vibrations and defects of the lattice.

\section{References}

[1] Pippard A.B. Philos. Mag., 1955, v. 46, 1115.

[2] Abrikosov A. A. Fundamentals of the Theory of Metals (Nauka, Moscow,1987; North-Holland, Amsterdam, 1998).

[3] Sondheimer E. H. The mean free path of electrons in metals // Advances in Physics, 2001, v. 50, No. 6, 499-537.

[4] Landau L.D., Lifshits E.M. Electrodynamics of Continuous Media, Butterworth-Heinemann (Jan 1984).

[5] Akhiezer A.I. , Kaganov M.I. and Liubarskii G.La. Ultrasonic Absorption in Metals//Soviet Physics (JETP). V. 5, 4. November, 1957, 685-688. 
[6] Rimlyand V. I., Drachev K. A. Acoustic wave propagation in metal pipes// The 22nd International Congress on Sound and Vibration, Florence (Italy), 12-16 July, 2015, 1 - 7.

[7] Bo Zhang Advances in sound absorption and propagation properties of popous metals under harsh environment conditions // Inter-Noise 2016, Hamburg, 4066 - 4072.

[8] Kotkin G.L. Ultrasonic attenuation in metals// J. Exptl. Theoret. Phys. (U.S.S.R.) v. 36, 1958, 941-942.

[9] Takimoto N. Dynamical Theory of Ultrasonic Attenuation In Metals // Progress of Theoretical Physics, v. 25, No. 3, March 1961, 327-352.

[10] Singh D., Bhalla V., Kumar R., Tripathi S. Behaviour of acoustical phonons in CeAs in low temperature region // Indian Journal of Pure and Applied Physis, v. 53, March, 2015, 169 - 174.

[11] Khan F.S., Auerbach A., Allen P.B. Sound Attenuation in Metals: Reformulation Of Pippard Theory to Include Microscopic Band Structure And Scattering// Solid State Communications, v.54, No.2 , 1985, $35-137$.

[12] Becker K., Fulde P., Keller J. Thalmeier P. Theory of ultrasonic attenuation in metallic rare earth systems // Journal de Physique Colloques, v. 40 (C5), 1979, C5-35-C5-37.

[13] Lüthi B. Physical Acoustics in the Solid State. Springer, BerlinHeidelberg-New York. Springer Series in solid-state sciences, 2007.

[14] De Gennaro S., Rettory A. The low-temperature electrical resistivity of potassium: size effects and the role of normal electron-electron scattering// J. Physics. F., v. 14, 1984, L237 - L242.

[15] De Gennaro S., Rettory A. Normal electron-electron contribution to the anomalous surface impedance // J. Phys. F. v. 15, 1985, L227 L230.

[16] Moiseev I. O., Yushkanov A. A. , Yalamov Yu. I. Calculation of the Electromagnetic Absorption by a Small Metal Particle with a TwoParameter Kinetic Equation// Optics and Spectroscopy, v.101. No.5, $2006797-801$. 
[17] Gall D. Electron mean free path in elemental metals// J. Appl. Phys., v. $119,2016,085101$.

[18] Aleksandrov A. F., Bogdankevich L. S., Rukhadze A. A. Principles of plasma electrodynamics. Springer-Verlag, 1984.

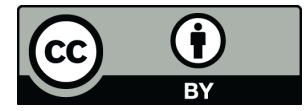

(C)2017 by the authors; licensee Preprints, Basel, Switzerland. This article is an open access article distributed under the terms and conditions of the Creative Commons Attribution (CC BY) license http://creativecommons.org/licenses/by/4.0/). 